\title{
Single-swab sampling for suspected COVID-19 cases: a time-dependent function?
}

\author{
Petros V. Vlastarakos ${ }^{1,4}\left(\right.$ Efterpi Michailidou $^{2} \cdot$ Dimitrios Katsianos $^{3}$ \\ Received: 29 July 2021 / Accepted: 2 August 2021 / Published online: 7 August 2021 \\ (c) The Author(s), under exclusive licence to Springer-Verlag GmbH Germany, part of Springer Nature 2021
}

Keywords COVID-19 $\cdot$ Infection $\cdot$ Swab $\cdot$ Sampling $\cdot$ Diagnosis

Dear Sirs,

SARS-CoV-2 is an enveloped, positive-sense, singlestranded RNA $\beta$-coronavirus. The resulting COVID-19 infection spread rapidly worldwide, and has become a public health emergency of international concern (pandemic). Although SARS-CoV-2 has a lower case-fatality rate, compared to previously described highly pathogenic human coronaviruses, such as MERS, or SARS-CoV-1, it spreads more efficiently, making it difficult to contain. All people are susceptible to SARS-CoV-2, including infants and children, and human-to-human transmission has been well established. Hence, the SARS-CoV-2 pandemic is not only an enormous burden to public health, stretching healthcare systems to their boundaries, but has already markedly affected civil societies, through the restrainment of human relationships, and the global economy from the imposed confinement in transports.

Rapid and accurate detection of SARS-CoV-2 nucleic acid in upper respiratory specimens is essential for COVID19 management, including diagnosis, risk assessment of transmission, and decisions regarding quarantine of patients [1]. Naso- and/or oropharyngeal swabs (NPS and OPS, respectively) have been used in this respect; however, potential differences in the detection rate and sensitivity of the two

Petros V. Vlastarakos

pevlast@hotmail.com; pevlast@yahoo.gr

1 ENT Department, MITERA Infirmary, 6 Erythrou Stavrou Str., 15123 Marousi-Athens, Greece

2 ENT Department, Bürger Spital, Solothurn, Switzerland

3 Department of Internal Medicine, MITERA Infirmary, Athens, Greece

4 Private Practice, 85 Ethnikis Antistaseos Str., 17236 Ag. Dimitrios-Athens, Greece sampling methods still represent issues of debate [1, 2]. In addition, real-life clinical practice dictates that testing prehospitalized individuals with both methods at the same time may not be cost-effective. In this context, single-swab sampling methods with high sensitivity towards SARS-CoV-2 detection are increasingly sought after.

The SARS-CoV-2 encodes, among other structural proteins, the spike protein, a type I glycoprotein, which protrudes from the surface of the virus, and binds to a metallopeptidase, called angiotensin-converting enzyme 2 (ACE2), which is subsequently employed as a receptor for target cell entry [3]. Upon binding ACE2, the spike protein of SARS$\mathrm{CoV}-2$ is cleaved by host proteases, thereby releasing the spike fusion peptide and facilitating host cell entry [4]. ACE2 has been detected in multiple epithelial cell types across the airway; the highest expression of ACE2 is found in nasal secretory (goblet) and ciliated cells [5]. Nevertheless, ACE2 is also expressed in the oral cavity [4], more in the tongue, than the buccal or gingival tissues. Since the viral attachment and entry are critical for replication and infection, the spatial distribution of ACE2 receptors could directly influence the diagnostic sensitivity of single-swab sampling methods.

Drawing on the sensitivity of NPS versus OPS, when performing SARS-CoV-2 sampling, and despite contradicting reports in the literature [1,2], ENT physicians should take into account that viral replication occurs promptly and abundantly, and the associated viral load is locally increased at an exponential degree, being influenced by both the temporal and spatial dimensions of the affected mucosa. That is to say that a narrow area (nose/nasopharynx vs. oropharynx), with lower overall volume of secretions (nasal discharge vs. saliva), and higher ACE2 expression may be more suitable for the diagnosis of mild or subclinical infections using single-swab sampling, in line with anecdotal reports from first-line medical personnel. History-taking may be a key 
element, in deciding the appropriate single-swab sampling method in terms of cost-effectiveness, as the temporal distribution of viral load seems to progress towards the lower airway, following the time of initial exposure to the virus.

Funding The authors have no financial interest and have not received any financial support for this article.

\section{Declarations}

Conflict of interest The authors have no conflicts of interest, and have not received any financial support for this article.

Ethical approval This article does not contain any studies with human participants or animals performed by any of the authors.

\section{References}

1. Wang H, Liu Q, Hu J et al (2020) Nasopharyngeal swabs are more sensitive than oropharyngeal swabs for COVID-19 diagnosis and monitoring the SARS-CoV-2 load. Front Med 7:334
2. Wyllie AL, Fournier J, Casanovas-Massana A et al (2020) Saliva or nasopharyngeal swab specimens for detection of SARS-CoV-2. N Engl J Med 383(13):1283-1286

3. Sungnak W, Huang N, Bécavin C et al (2020) SARS-CoV-2 entry factors are highly expressed in nasal epithelial cells together with innate immune genes. Nat Med 26(5):681-687

4. Srinivasan M, Zunt S, Goldblatt L (2020) Oral epithelial expression of angiotensin converting enzyme-2: implications for COVID-19 diagnosis and prognosis. https://doi.org/10.1101/2020. 06.22 .165035

5. Luan J, Lu Y, Jin X, Zhang L (2020) Spike protein recognition of mammalian ACE2 predicts the host range and an optimized ACE2 for SARS-CoV-2 infection. Biochem Biophys Res Commun 526(1):165-169

Publisher's Note Springer Nature remains neutral with regard to jurisdictional claims in published maps and institutional affiliations. 